ISSN 2525-4804

\title{
TEMPERATURAS E SUBSTRATOS PARA O TESTE DE GERMINAÇÃO DE SEMENTES DE Cassia leptophylla Vogel
}

\author{
Matheus Santin Padilha ${ }^{1}$ Marcelo Mocelin Parcianello ${ }^{1}$, Alex Sandro Moraes de Oliveira ${ }^{1}$, Lúcia \\ Salengue Sobral ${ }^{2}$
}

\section{RESUMO}

O teste de germinação é o principal parâmetro de avaliação da qualidade fisiológica de sementes. Entretanto, para a maioria das espécies florestais nativas ainda há uma grande falta de informação sobre a condução do teste de germinação, o que dificulta a elaboração de protocolos para avaliação da qualidade fisiológica. Diante disso, o objetivo do trabalho foi avaliar diferentes temperaturas e substrato para a realização do teste de germinação de sementes de Cassia leptophylla. O delineamento experimental utilizado foi inteiramente casualizado, em arranjo fatorial 4x4, sendo quatro substratos (areia, papel mata borrão, papel toalha e vermiculita) e quatro temperaturas $\left(20,25,30\right.$ e $\left.20-30^{\circ} \mathrm{C}\right)$ com quatro repetições de 50 sementes. As variáveis analisadas foram: plântulas normais, primeira contagem de germinação, índice de velocidade de germinação e tempo médio de germinação. A temperatura constante de $30{ }^{\circ} \mathrm{C}$ em substrato areia e a temperatura alternada $20-30{ }^{\circ} \mathrm{C}$ em substrato papel toalha são as condições que favorecem a porcentagem e velocidade de germinação, sendo indicadas para a condução do teste de germinação de C. leptophylla.

Palavras-chave: análise de sementes, qualidade fisiológica, sementes florestais.

\section{TEMPERATURE AND SUBSTRATES FOR Cassia leptophylla Vogel SEEDS GERMINATION TEST}

\section{ABSTRACT}

The germination test is the main parameter for evaluating the physiological quality of seeds. However, for the most native forest species there is still a great lack of information about the conduct of the germination test, this makes difficult to prepare protocols for physiological quality evaluation. Therefore, the objective of this work was to evaluate different temperature and substrate for Cassia leptophylla seeds germination test. The experimental design was completely random, in 4x4 factorial arrangements, with four substrates (sand, blotting paper, paper towels and vermiculite) and four temperatures $\left(20,25,30\right.$ and 20-30 $\left.{ }^{\circ} \mathrm{C}\right)$ with four replications of 50 seeds. The variables analyzed were: normal seedlings, first germination count, germination speed index and average germination time. The constant temperature of $30{ }^{\circ} \mathrm{C}$ in substrate sand and the temperature $20-30{ }^{\circ} \mathrm{C}$ in substrate paper towel are the conditions that favor the percentage and germination speed, these conditions are indicated for conduction of the $C$. leptophylla germination test.

Keywords: seed analysis, physiological quality, forest seeds.

\footnotetext{
${ }^{1}$ Graduando em Agronomia; Universidade Comunitária da Região de Chapecó - UNOCHAPECÓ; Chapecó - SC; matheus_santin@hotmail.com; parcianello@unochapeco.edu.br; aleques.div@ hotmail.com.

${ }^{2}$ Engenheira Agrônoma, Mestre em Agronomia; Universidade Comunitária da Região de Chapecó - UNOCHAPECÓ; Chapecó SC; luciass@unochapeco.edu.br.
} 


\section{INTRODUÇÃO}

Cassia leptophylla Vogel é uma espécie florestal nativa da família Fabaceae, conhecida popularmente como grinalda-de-noiva, falsobarbatimão, medalhão-de-ouro, entre outros. Ocorre no bioma Mata Atlântica, sendo encontrada no estado de São Paulo e região sul do Brasil. A espécie é amplamente utilizada para arborização urbana, reflorestamentos e recuperação de áreas degradadas (Carvalho, 2008).

Para a produção de mudas de espécies florestais é de grande importância o uso de sementes de qualidade buscando otimizar a produção destas em viveiros florestais. Gomes et al. (2016) relatam que para determinar o potencial fisiológico de um lote de sementes e comparar a diferença entre eles utiliza-se o teste de germinação, que é efetuado em condições ótimas de temperatura, substrato, luz e umidade, de maneira a permitir que a semente germine de forma regular e rápida. As interações entre esses fatores são objeto de estudo de diversas pesquisas relacionadas à determinação de métodos para a avaliação da qualidade fisiológica de sementes.

O substrato fornece suporte físico para as sementes, fornecendo condições adequadas de água e oxigênio através de sua capacidade de retenção hídrica e de aeração. Os principais substratos indicados para condução do teste de germinação pelas Regras de Análise de Sementes (RAS) são o papel (toalha, filtro e mata-borrão) e a areia (Brasil, 2009). No entanto, especificamente para sementes de espécies florestais a vermiculita também é empregada como substrato para diversas espécies, devido à sua alta capacidade de retenção de água e de sua baixa densidade (Brasil, 2013).

As temperaturas de $25^{\circ} \mathrm{C}$ e $30^{\circ} \mathrm{C}$ são as que favorecem a germinação de espécies arbóreas. Contudo, para algumas sementes florestais o uso de temperaturas alternadas é o mais recomendado (Brancalion et al., 2010), como verificado para Peltophorum dubium (Alves et al., 2011) e Caesalpinia pyramidalis (Lima et al., 2011), Cedrela fissilis (Oliveira e Barbosa, 2014), Campomanesia xanthocarpa (Gomes et al., 2016) e Parkia platycephala (Silva et al., 2017).

As RAS (Brasil, 2009) possuem procedimentos para a condução do teste de germinação de espécies cultivadas e algumas espécies florestais. Entretanto, em relação às espécies florestais, existe ainda uma lacuna devido à falta de informações sobre a germinação e outras características fisiológicas das sementes, o que dificulta a elaboração de manuais de controle de qualidade (Wielewicki et al., 2006).

As metodologias existentes para realização do teste de germinação de sementes de espécies florestais são incipientes se comparadas às das espécies agrícolas. Entretanto, a partir do conhecimento gerado pelas pesquisas foram elaboradas as Instruções para Análise de Sementes Florestais (Brasil, 2013), as quais fornecem recomendações para a condução do teste de germinação de diversas espécies florestais. Porém, para várias espécies, há necessidade de aperfeiçoamento das metodologias sugeridas.

Neste contexto, o objetivo deste trabalho foi avaliar o substrato e a temperatura adequada para a condução do teste de germinação de sementes de $C$. leptophylla.

\section{MATERIAL E MÉTODOS}

Os frutos de C. leptophylla foram coletados em um remanescente florestal localizado entre as coordenadas $27^{\circ} 10^{\prime} 40^{\prime \prime}$ S e 52 $39^{\circ} 35^{\prime \prime}$ O, no Distrito de Marechal Bormann, Chapecó, Santa Catarina. A coleta foi efetuada em sete árvores matrizes, e conforme proposto por Carvalho (2008), quando apresentavam coloração escura. Após a coleta as sementes foram extraídas dos frutos com o auxílio de um alicate e beneficiadas para a eliminação das impurezas, sementes imaturas e danificadas.

$\mathrm{O}$ experimento foi realizado em delineamento experimental inteiramente casualizado em arranjo fatorial $4 \times 4$ (temperatura $x$ substrato) com quatro repetições de 50 sementes.

O teste de germinação foi conduzido em 
germinadores tipo Biochemical Oxigen Demand (BOD) nas temperaturas constantes de $20,25,30{ }^{\circ} \mathrm{C}$ e alternada de $20-30{ }^{\circ} \mathrm{C}(12 \mathrm{~h}-12 \mathrm{~h})$. A semeadura foi realizada em papel toalha na forma de rolo (RP), acondicionados em saco plástico transparente para evitar a perda de água por evaporação; sobre papel mata-borrão (SP), sobre areia (SA) e sobre vermiculita $(\mathrm{SV})$, todos em caixas plásticas tipo gerbox $(10,5 \mathrm{~cm} \times 10,5 \mathrm{~cm} \times 3,0 \mathrm{~cm})$. A areia e a vermiculita foram previamente esterilizados em estufa a $120^{\circ} \mathrm{C}$ por $12 \mathrm{~h}$.

O umedecimento dos substratos foi realizado no início do teste, utilizando-se para a areia um volume de água correspondente a $60 \%$ da capacidade de campo; para a vermiculita 1,5 vezes o de sua massa seca em água, para o papel toalha e para o papel mataborrão utilizou-se 2,5 vezes o peso seco do papel (Brasil, 2013).

Antes da semeadura realizou-se a superação da dormência das sementes através da imersão em ácido sulfúrico $\left(\mathrm{H}_{2} \mathrm{SO}_{4}\right)$ concentrado $(95-97 \%)$ por 20 min (Wielewick et al., 2006), seguida de lavagem em água destilada e secagem sobre papel toalha por 10 min. Na sequência, as sementes foram tratadas com fungicida Captan SC $®$ na dose de $250 \mathrm{~mL}$ do produto comercial/100 kg de sementes, conforme recomendação do fabricante para espécies cultivadas. Para a avaliação do efeito da interação temperatura $\mathrm{x}$ substrato foram analisadas as variáveis: germinação (plântulas normais), primeira contagem de germinação (PCG), índice de velocidade de germinação (IVG) e tempo médio de germinação (TMG).

A primeira contagem de germinação (PCG) foi representada pelo número de plântulas normais obtidas no $7^{\circ}$ dia após a semeadura. A germinação correspondeu à porcentagem de plântulas normais obtidas até os 20 dias após a semeadura e foram caracterizadas como aquelas que apresentaram todas as estruturas essenciais desenvolvidas, raiz primaria, hipocótilo, epicótilo, cotilédone e plúmula (Brasil, 2009).

O IVG foi calculado através do somatório do número de plântulas normais formadas a cada dia
(Gi), dividido pelo número de dias decorridos a partir da semeadura (Ni), conforme proposto por Maguire (1962):

$$
\mathrm{IVG}=\sum(\mathrm{Gi} / \mathrm{Ni})
$$

Para o cálculo do TMG utilizou-se o número de plântulas normais contabilizadas no intervalo entre cada contagem $\left(\mathrm{n}_{\mathrm{i}}\right)$ e o tempo decorrido entre o início da germinação e a i-ésima contagem $\left(\mathrm{t}_{\mathrm{i}}\right)$ conforme proposto por Labouriau (1983):

$$
\mathrm{TMG}=\sum\left(\mathrm{n}_{\mathrm{i}} \mathrm{t}_{\mathrm{i}}\right) /\left(\sum \mathrm{n}_{\mathrm{i}}\right)
$$

Os resultados obtidos foram submetidos à análise de variância e as médias comparadas pelo teste de Tukey a $5 \%$ de probabilidade. A análise foi realizada através do programa computacional Assistat versão 7.7 (Silva e Azevedo, 2016).

\section{RESULTADOS E DISCUSSÃO}

A análise dos dados demonstrou interação significativa $(p<0,05)$ entre os fatores (substratos $\mathrm{x}$ temperaturas) para a variável germinação, primeira contagem de germinação (PCG), índice de velocidade de germinação (IVG) e tempo médio de germinação (TMG) (Tabela 1).

Os resultados referentes à porcentagem de plântulas normais (germinação) de $C$. leptophylla demonstram que o tratamento de superação de dormência foi eficiente, visto que em diversas combinações de tratamentos foi verificada porcentagem de germinação superior a $90 \%$, independente do substrato ou da temperatura. Essa afirmação indica que os efeitos observados nas variáveis foram causados pela combinação dos tratamentos utilizados (Tabela 2).Para a temperatura de 20,30 e $20-30{ }^{\circ} \mathrm{C}$ observa-se que no papel mataborrão a germinação foi significativamente inferior aos demais substratos, indicando que este substrato não foi adequado para a germinação de sementes de C. leptophylla, quando associado a estas temperaturas (Tabela 2). De acordo com Alves et al. (2014), um 
dos fatores que contribuem para a germinação inferior no papel mata-borrão é a menor área de contato da semente com o substrato que pode influenciar a porcentagem total de germinação.

Tabela 1 - Resumo do quadro de análise de variância das variáveis avaliadas

\begin{tabular}{cccccc}
\hline \multirow{2}{*}{ Fonte de Variação } & & \multicolumn{3}{c}{ Fc } \\
\cline { 3 - 6 } & GL & G & PCG & IVG & TMG \\
\hline Substrato $(\mathrm{S})$ & 3 & $52,261^{*}$ & $193,73^{*}$ & $120,752^{*}$ & $241,120^{*}$ \\
Temperatura $(\mathrm{T})$ & 3 & $19,275^{*}$ & $269,92^{*}$ & $45,724^{*}$ & $443,698^{*}$ \\
Interação $(\mathrm{S} * \mathrm{~T})$ & 9 & $9,093^{*}$ & $62,406^{*}$ & $11,261^{*}$ & $19,604^{*}$ \\
Erro & 48 & & & & \\
Total & 63 & & & & \\
\hline
\end{tabular}

G: germinação; PCG: Primeira contagem de germinação; IVG: índice de velocidade de germinação; TMG: tempo médio de germinação; Fc: F calculado; GL: Graus de liberdade; *, **Significativo a $1 \%$ e 5\%, respectivamente, pelo teste F.

Tabela 2 - Porcentagem média de plântulas normais de sementes de Cassia leptophylla submetidas à germinação em diferentes temperaturas e substratos

\begin{tabular}{lcccc}
\hline \multirow{2}{*}{ Substrato } & \multicolumn{4}{c}{ Temperatura $\left({ }^{\mathbf{0}} \mathbf{C}\right)$} \\
\cline { 2 - 4 } & $\mathbf{2 0}$ & $\mathbf{2 5}$ & $\mathbf{3 0}$ & $\mathbf{2 0 - 3 0}$ \\
\hline Areia & $87 \mathrm{Aa}^{*}$ & $92 \mathrm{Aa}$ & $93 \mathrm{Aa}$ & $87 \mathrm{Aa}$ \\
Vermiculita & $93 \mathrm{Aa}$ & $93 \mathrm{Aa}$ & $83 \mathrm{ABa}$ & $90 \mathrm{Aa}$ \\
Papel mata-borrão & $47 \mathrm{Bbc}$ & $92 \mathrm{Aa}$ & $40 \mathrm{Cc}$ & $58 \mathrm{Bb}$ \\
Papel toalha & $94 \mathrm{Aa}$ & $91 \mathrm{Aa}$ & $73 \mathrm{Bb}$ & $89 \mathrm{Aa}$ \\
\hline
\end{tabular}

C.V. $(\%)$

8,19

*Médias seguidas da mesma letra, minúscula na linha e maiúscula na coluna, não diferem estatisticamente entre si pelo teste de Tukey a $5 \%$ de probabilidade.

Por outro lado, as temperaturas de $20^{\circ} \mathrm{C}$ e 20 $30{ }^{\circ} \mathrm{C}$ aliadas aos substratos areia, vermiculita e papel toalha permitiram a obtenção de porcentagens de germinação entre 89 e 94\%, que não diferiram entre si. Todavia, referente à temperatura de $30^{\circ} \mathrm{C}$, a germinação foi superior na areia e vermiculita $(93 \%$ e $83 \%$, respectivamente), diferindo significativamente do papel toalha (73\%).

Lima et al. (2011) também verificaram que a temperatura de $30{ }^{\circ} \mathrm{C}$ e o substrato papel toalha influenciaram negativamente a germinação de sementes Caesalpinia pyramidalis e que as temperaturas de 20,25 e $20-30^{\circ} \mathrm{C}$ associadas aos substratos areia e vermiculita foram as melhores condições para o teste de germinação desta espécie (Tabela 2).

$\mathrm{O}$ teste de germinação conduzido em temperatura de $25{ }^{\circ} \mathrm{C}$ indicou que a porcentagem média de plântulas normais variou entre 91 e 93\%, não havendo diferença significativa entre os substratos utilizados. Resultado similar foi encontrado por Santos et al. (2015) para Cedrela fissilis na temperatura de $25{ }^{\circ} \mathrm{C}$, na qual a porcentagem de germinação foi superior a $90 \% \mathrm{em}$ todos os substratos testados. Para a maioria das espécies do Bioma Mata Atlântica, a temperatura ótima para a germinação é de $25^{\circ} \mathrm{C}$ (Brancalion et al., 2010). Esse fator pode explicar a germinação elevada de $C$. leptophylla para essa temperatura em todos os 
substratos, sendo essa uma alternativa de uso independente do substrato utilizado no estudo (Tabela 2).

Para a variável PCG a maior porcentagem de plântulas normais foi obtida na temperatura alternada de $20-30{ }^{\circ} \mathrm{C}$ em substrato papel toalha (68\%) e na temperatura constante de $30{ }^{\circ} \mathrm{C}$ em substrato areia (49\%) (Tabela 3).

A PCG é uma forma de determinar o vigor de diferentes lotes de sementes, em que lotes com maior vigor apresentam maior porcentagem de primeira contagem de germinação (Carvalho e Nakagawa, 2012). No substrato papel mata-borrão não foi verificada plântulas normais na primeira contagem de germinação, independente da temperatura utilizada. Segundo Alves et al. (2014) a velocidade da germinação é afetada pelo contato do substrato com as sementes, sendo o mesmo observado anteriormente para a porcentagem de germinação com este substrato. Os resultados com a temperatura de $25{ }^{\circ} \mathrm{C}$ demonstram que o substrato papel toalha apresentou a maior PCG, indicando que os substratos areia, vermiculita e papel mata borrão retardaram a velocidade de germinação das sementes de $C$. leptophylla (Tabela 3).

Tabela 3 - Porcentagem média de plântulas normais obtidas na primeira contagem de germinação (PCG) de sementes de Cassia leptophylla submetidas à germinação em diferentes temperaturas e substratos

\begin{tabular}{lcccc}
\hline \multirow{2}{*}{ Substrato } & \multicolumn{4}{c}{ Temperatura $\left({ }^{\circ} \mathbf{C}\right)$} \\
\cline { 2 - 4 } & $\mathbf{2 0}$ & $\mathbf{2 5}$ & $\mathbf{3 0}$ & $\mathbf{2 0 - 3 0}$ \\
\hline Areia & $0 \mathrm{Ac} c^{*}$ & $0 \mathrm{Bc}$ & $49 \mathrm{Aa}$ & $28 \mathrm{Bb}$ \\
Vermiculita & $0 \mathrm{Ab}$ & $0 \mathrm{Bb}$ & $14 \mathrm{Ca}$ & $19 \mathrm{Ca}$ \\
Papel mata-borrão & $0 \mathrm{Aa}$ & $0 \mathrm{Ba}$ & $0 \mathrm{Da}$ & $0 \mathrm{Da}$ \\
Papel toalha & $0 \mathrm{Ac}$ & $14 \mathrm{Ab}$ & $19 \mathrm{Bb}$ & $68 \mathrm{Aa}$ \\
\hline
\end{tabular}

C.V. $(\%)$

22,78

* Médias seguidas da mesma letra, minúscula na linha e maiúscula na coluna, não diferem estatisticamente entre si pelo teste de Tukey a $5 \%$ de probabilidade.

A temperatura é um fator que afeta a porcentagem, a velocidade e a uniformidade de germinação, uma vez que controla as reações bioquímicas que regulam todo o processo metabólico da germinação (Marcos-Filho, 2015) e, geralmente, temperaturas mais altas aceleramos processos metabólicos e temperaturas mais baixas retardam os processos (Bewley et al., 2013). Dessa forma, a menor porcentagem de PCG observada nas temperaturas de 20 e $25^{\circ} \mathrm{C}$ é resultado do efeito da temperatura utilizada no teste. Corroborando com os resultados, Alves et al. (2011) obtiveram para sementes de Peltophorum dubium porcentagem de PCG superior nos substratos areia e papel toalha quando associados às temperaturas de $30^{\circ} \mathrm{C}$ e $20-30$ ${ }^{\circ} \mathrm{C}$ e, com o uso de substrato papel mata-borrão em temperaturas constantes de $25,30,35^{\circ} \mathrm{C}$ e alternada de $20-30{ }^{\circ} \mathrm{C}$ verificaram que a PCG foi nula.

Para os resultados de IVG e TMG (Tabela 4) observa-se que as interações temperatura constante de $30^{\circ} \mathrm{C}$ com o substrato areia e a temperatura alternada $20-30^{\circ} \mathrm{C}$ em substrato papel toalha apresentaram maior IVG $(6,86)$ e menor TMG (6,62 dias), corroborando os resultados de primeira contagem (Tabela 3).

Com exceção do tratamento utilizando o substrato papel mata-borrão, a temperatura alternada de $20-30{ }^{\circ} \mathrm{C}$ proporcionou maior IVG em relação às 
temperaturas de $20^{\circ} \mathrm{C}$ e $25^{\circ} \mathrm{C}$ (Tabela 4). Isso ocorre porque as temperaturas alternadas favorecem, principalmente, a germinação de espécies que não passaram por intenso processo de domesticação (Marcos-Filho, 2015) entre essas, as espécies florestais, já que as temperaturas alternadas simulam as condições que ocorrem no solo (Oliveira e Barbosa, 2014). Semelhante ao obtido no presente estudo, o papel toalha em temperatura alternada 20 $30^{\circ} \mathrm{C}$ apresentou o maior resultado de IVG para Peltophorum dubium (Alves et al., 2011) e para $P$. platycephala (Bezerra et al., 2017).

Tabela 4 - Resultados do índice de velocidade de germinação (IVG) e tempo médio de germinação (TMG) de sementes de Cassia leptophylla submetidas à germinação em diferentes temperaturas e substratos

\section{Temperatura $\left({ }^{\circ} \mathrm{C}\right)$}

Substratos

2025

30

20-30

\begin{tabular}{lcccc} 
& \multicolumn{3}{c}{ IVG } \\
\cline { 2 - 5 } Areia & $3,52 \mathrm{Bc}^{*}$ & $4,73 \mathrm{ABb}$ & $6,38 \mathrm{Aa}$ & $5,60 \mathrm{Bab}$ \\
Vermiculita & $3,94 \mathrm{Bc}$ & $4,56 \mathrm{BCbc}$ & $5,01 \mathrm{Bab}$ & $5,61 \mathrm{Ba}$ \\
Papel mata-borrão & $1,26 \mathrm{Cc}$ & $3,76 \mathrm{Ca}$ & $2,12 \mathrm{Cb}$ & $3,10 \mathrm{Ca}$ \\
Papel toalha & $4,83 \mathrm{Abc}$ & $5,58 \mathrm{Ab}$ & $4,40 \mathrm{Bc}$ & $6,86 \mathrm{Aa}$ \\
\hline \multicolumn{1}{c}{ C.V.(\%) $\quad$} & & $\mathbf{1 0 , 5 2}$ & \\
\hline \multicolumn{1}{c}{ Substratos } & & $\mathbf{T M G}$ & $7,93 \mathrm{Bc}$ \\
Areia $\quad 12,53 \mathrm{Ba} *$ & $9,76 \mathrm{Bb}$ & $7,44 \mathrm{Cc}$ & $8,10 \mathrm{Bc}$ \\
Vermiculita & $11,93 \mathrm{Ca}$ & $10,24 \mathrm{Bb}$ & $8,43 \mathrm{Bc}$ & $9,65 \mathrm{Ac}$ \\
Papel mata-borrão & $15,12 \mathrm{Aa}$ & $12,39 \mathrm{Ab}$ & $9,62 \mathrm{Ac}$ & $6,62 \mathrm{Cc}$ \\
Papel toalha & $9,79 \mathrm{Da}$ & $8,18 \mathrm{Cb}$ & $8,44 \mathrm{Bb}$ & \\
\hline
\end{tabular}

\section{C.V. $(\%)$}

3,77

*Médias seguidas da mesma letra, minúscula na linha e maiúscula na coluna, não diferem estatisticamente entre si pelo teste de Tukey a 5\% de probabilidade.

Para o substrato areia a temperatura de $30{ }^{\circ} \mathrm{C}$ apresentou o maior IVG $(6,38)$ e menor TMG $(7,44)$ em relação às demais temperaturas (Tabela 4). Azerêdo et al. (2011) destacam que a velocidade de germinação é favorecida por temperaturas um pouco mais altas. Dessa forma, resultados semelhantes foram obtidos para Caesalpinia ferrea (Lima et al., 2006) e para Ormosia arborea (Oliveira et al. 2016), em que, o uso da temperatura de $30{ }^{\circ} \mathrm{C}$ com o substrato areia adiantou o processo de germinação, proporcionando o maior IVG e o menor TMG. Os parâmetros associados à velocidade de germinação são desejados durante os testes em laboratório. Nesse sentido, mesmo que o uso de areia em laboratórios seja dificultoso dependendo da estrutura disponível, esse substrato proporciona plasticidade no planejamento das análises em virtude do menor tempo necessário.

Para o substrato papel mata-borrão o IVG foi o menor e o TMG foi o maior observado em todas as 
temperaturas utilizadas (Tabela 4). Esse resultado também foi constatado por Alves et al. (2011) em que sementes de Peltophorum dubium tiveram a sua germinação prejudicada no substrato papel mataborrão, apresentando o menor IVG nas temperaturas constantes de $25,30,35^{\circ} \mathrm{C}$ e alternada $20-30{ }^{\circ} \mathrm{C}$. O uso de papel mata borrão em condição de sobre papel não foi favorável devido ao menor contato $\mathrm{e}$ disponibilidade de água para as sementes, principalmente quando combinado com a temperatura de $20^{\circ} \mathrm{C}$. Entretanto, essa informação se torna importante ao identificar a amplitude e necessidades da espécie. Considerando isso, o uso do substrato mata borrão em temperatura de $20^{\circ} \mathrm{C}$ pode ser viável para outras tipos de testes, como os testes de sanidade.

$\mathrm{Na}$ temperatura de $20{ }^{\circ} \mathrm{C}$ foi verificada alta porcentagem final de plântulas normais nos substratos areia, vermiculita e papel toalha (Tabela 2). Contudo, para esta temperatura, nota-se o menor IVG e o maior TMG para todos os substratos utilizados (Tabela 4). A germinação em $20{ }^{\circ} \mathrm{C}$ foi lenta, aumentando o tempo de duração dos testes. As temperaturas abaixo da ótima influenciam na velocidade de hidratação e das reações metabólicas, atrasando o processo germinativo (Bewley et al., 2013). A menor velocidade de germinação na temperatura de $20{ }^{\circ} \mathrm{C}$ também foi observada para Peltophorum dubium (Pereira et al., 2013), Parkia multijuga (Rocha et al., 2014) e, Ormosia arborea (Oliveira et al., 2016). A partir disso, é possível ressaltar que a $C$. leptophylla possui capacidade de germinação entre as temperaturas de 20 a $30{ }^{\circ} \mathrm{C}$, contudo, o sucesso da porcentagem final de plântulas é dependente do substrato utilizado.

Os resultados obtidos divergem dos dados apresentados nas Instruções para Análise de Sementes de Espécies Florestais (2013), a qual indica a temperatura de $25^{\circ} \mathrm{C} \mathrm{em}$ substrato papel toalha para o teste de germinação de sementes de C. leptophylla. No entanto, verificou-se que a temperatura de $25{ }^{\circ} \mathrm{C}$ em substrato papel toalha propiciou menor porcentagem de PCG (Tabela 3), menor IVG (Tabela 4) e maior TMG (Tabela 4), em relação às condições de temperatura alternada $20-30{ }^{\circ} \mathrm{C}$ com os substratos areia, papel toalha e vermiculita, assim como, quando se utilizou a temperatura constante de $30{ }^{\circ} \mathrm{C}$ com substrato areia. A partir disso, a presente pesquisa demonstrou as diferenças na germinação de $C$. leptophylla para diferentes condições, sendo possível a tomada de decisão para a escolha do procedimento baseado no material e estrutura disponível.

\section{CONCLUSÕES}

Diante dos resultados obtidos, verificou-se que as melhores condições para a condução de teste de germinação de sementes de $C$. leptophylla são: temperatura constante de $30{ }^{\circ} \mathrm{C}$ em substrato areia e temperatura alternada $20-30{ }^{\circ} \mathrm{C}$ em substrato papel toalha.

\section{AGRADECIMENTOS}

Os autores agradecem a Universidade Comunitária da Região de Chapecó (Unochapecó) por ceder o espaço e o material para realização da pesquisa, e ao Viveiro Florestal Universitário da Unochapecó pelo auxílio na coleta das sementes utilizadas.

\section{REFERÊNCIAS BIBLIOGRÁFICAS}

Alves, E.U.; Guedes, R.S.; Gonçalves, E.P.; Viana, J.S.; Santos, S.S. \& Moura, M.F. (2011). Effect of temperature and substrate on germination of Peltophorum dubium (Sprengel) Taubert seeds. Acta Scientiarum Biological Sciences, 33(1): 113-118. https://doi.org/10.4025/actascibiolsci.v33i1.7057.

Alves, E.U.; Santos-Moura, S.S.; Moura, M.F.; Guedes, R.S. \& Estrela, F.A. (2012). Germinação e vigor de sementes de Crataeva tapia L. em diferentes substratos temperaturas. Revista Brasileira de Fruticultura, $\quad 34(4)$ : 1208-1215. https://doi.org/10.1590/S0100-29452012000400030.

Alves, C.Z.; Candido, A.C.S.; Oliveira, N.C. \& Lourenço, F.M.S. (2014). Teste de germinação em sementes de Cucumis metuliferus E. Mey. Ciência 
Rural,

44(2):

228-234.

(Embrapa Florestas. Circular Técnica 151).

https://doi.org/10.1590/S0103-84782014000200006.

Araújo, A.M.S.; Assis, L.C.S.L.C.; Nogueira, N.W.; Freitas, R.M.O. \& Torres, S.B. (2016). Substrates and temperatures for the germination of seeds of Senegalia tenuifolia (L.) BRITTON \& ROSE. Revista Caatinga, 29(1), 113-118. http://dx.doi.org/10.1590/1983-21252016v29n113rc.

Azeredo, G.A.; Paula, R.C. \& Valeri, S.V. (2011). Temperatura e substrato para a germinação de sementes de Piptadenia moniliformis Benth. Scientia Forestalis, 39(92): 479-488.

Bassaco, M.V.M.; Nogueira, A.C. \& Cosmo, N.L. (2014). Avaliação da germinação em diferentes temperaturas e substratos e morfologia do fruto, semente e plântula de Sebastiania brasiliensis. Floresta, $\quad 44(3)$ : 381-392. http://dx.doi.org/10.5380/rf.v44i3.32076.

Bezerra, R.; Matos, V.P.; Farias, S.G.G., Sena, L.H.D.M. \& Silva, D. Y. B. D. (2017). Germinação e vigor de plântulas de Parkia platycephala Benth. em diferentes substratos e temperaturas. Revista Ciência Agronômica, 48(1): 142-150. https://doi.org/10.5935/1806-6690.20170016.

Bewley, J.D.; Braford, K.J.; Hilhorst, H.W.M. \& Nonogaki, H. Seeds: Physiology of development, germination and dormancy. $3^{\mathrm{a}}$ ed. New York: Springer, 2013. 392p.

Brancalion, P.H.S.; Novembre, A.D.L.C. \& Rodrigues, R.R. (2010). Temperatura ótima de germinação de sementes de espécies arbóreas brasileiras. Revista Brasileira de Sementes, Londrina, 32(4): 15-21. https://doi.org/10.1590/S0101-31222010000400002.

Brasil. Ministério da Agricultura e Reforma Agrária. (2009) Regras para Análise de Sementes. Departamento Nacional de Defesa Vegetal. Brasília, $395 \mathrm{p}$.

Brasil. Ministério da Agricultura, Pecuária e Abastecimento. (2013) Instruções para Análise de Sementes de Espécies Florestais. Brasília. 97p.

Carvalho, P.E.R. (2008) Grinalda-de-noiva (Cassia Disponível em: $<$ https://ainfo.cnptia.embrapa.br/digital/bitstream/C NPF-2009-09/44064/1/circ-tec151.pdf $>$.

Carvalho, N.M. \& Nakagawa, J. (2012). Sementes: ciência tecnologia e produção. $4^{\text {a }}$ ed. Jaboticabal: FUNEP. 429 p.

Dutra, A.F.; Araujo, M.M.; Rorato, D.G. \& Mieth, P. (2016) Germinação de sementes e emergência de plântulas de Luehea divaricata Mart. Et. Zucc. em diferentes substratos. Ciência Florestal, 26(2): 411 418. http://dx.doi.org/10.5902/1980509822744.

Figliolia, M.B. (2015a) A pesquisa e o estabelecimento de técnicas para análise de sementes florestais no Brasil. In: PIÑARODRIGUES, F.C.M; FIGLIOLIA, M.B \& SILVA, A. Sementes Florestais Tropicais: da ecologia à produção. Londrina: ABRATES, 286-288 p.

Figliolia, M.B. (2015b) Teste de Germinação. In: Piña-Rodrigues, F.C.M; Figliolia \& M.B; Silva, A. Sementes Florestais Tropicais: da ecologia à produção. Londrina: ABRATES, 325-343 p.

Gomes, J.P.; Oliveira, L.M.; Ferreira, P.I.; Batista, F.F. (2016). Substratos e temperaturas para teste de germinação em sementes de Myrtaceae. Ciência Florestal, 26(4): 285-293. http://dx.doi.org/10.5902/1980509821120.

Laboriau, L.G. (1983) A Germinação de Sementes. Washington: OEA. 174p.

Lima, C.R.; Pacheco, M.V.; Bruno, R.L.A.; Ferrari, C.S.; Braga-Júnior, J.M. \& Bezerra, A.K.D. (2011). Temperaturas e substratos na germinação de sementes de Caesalpinia pyramidalis TUL. Revista Brasileira de Sementes, 33(2): 216-222. https://doi.org/10.1590/S0101-31222011000200003.

Lima, J.D.; Almeida, C.C.; Dantas, V.A.V.; Silva, B.M.S. \& Moraes, W.S. (2006). Efeito da temperatura e do substrato na germinação de sementes de Caesalpinia ferrea mart. ex tul. (Leguminosae, Caesalpinoideae). Revista Árvore, 30(4): 513-518. https://doi.org/10.1590/S0100$\underline{67622006000400003}$.

leptophylla). Colombo: Embrapa Florestas. 6 p. 
Maguire, J.D. (1962). Speed of germination-aid in selection and evaluation for seedling emergence and vigor. Crop Science, 2(1): 176-177.

Oliveira, F.N.; França, F.D.; Torres, S.B.; Nogueira, N.W. \& Freitas, R.M.O. (2016). Temperaturas e substratos na germinação de sementes de pereiro vermelho (Simira gardneriana M.R. Barbosa \& Peixoto). Revista Ciência Agronômica, 47(4): 658666. https://doi.org/10.5935/1806-6690.20160079.

Oliveira, A.K.M. \& Barbosa, L.A. (2014). Efeitos da temperatura na germinação de sementes e na formação de plântulas de Cedrela fissilis. Floresta, 44(3): 441-450.

http://dx.doi.org/10.5380/rf.v44i3.33260.

Oliveira, A.K.M.; Carvalho, J.M.B.; Souza, J.S. \& Souza, S.A. (2015). Germinação de sementes de Aspidosperma subincanum Mart. ex A. DC em diferentes temperaturas. Revista Brasileira de Plantas medicinais, (17)4: 642-648. https://doi.org/10.1590/1983-084X/14_082.

Oliveira, A. K. M.; Souza, J.S.; Carvalho, J.M.B.; Souza, S.A. \& Bocchese, R.A. (2016). Germinação de sementes e crescimento de Ormosia arborea em diferentes temperaturas e substratos. Gaia Scientia, 10(4): 262-271. http://dx.doi.org/10.21707/gs.v10.n04a21.

Pereira, K.T.O.; Santos, B.R.V.; Benedito, C.P.; Lopes, E.G. \& Aquino, G.S.M. (2015). Germinação e vigor de sementes de Moringa oleífera Lam. em diferentes substratos e temperaturas. Revista Caatinga, 28(2): 92-99.

Pereira, S.R.; Kalife, C.; Rodrigues, A.P.D.C. \& Laura, V.A. (2013). Influência da temperatura na germinação de sementes de Peltophorum dubium (Spreng.) Taub. Informativo Abrates, 23(3): p.5255.

Rocha, C.R.M.; Costa, D.S.; Novembre, A.D.L.C. \& Cruz, E. D. (2014). Morfobiometria e germinação de sementes de Parkia multijuga Benth. Nativa, 2(1): 42-47.

Santos, S.R.G.; Bezerra, A.C.F.; Oliveira, P.C.F.; Silva, S.D.S. \& Paula, R.C. (2015). Avaliação de substratos e temperaturas para sementes de espécies florestais. Revista da Universidade Vale do Rio Verde, 13(1): 66-76.

Silva, F.A.S. \& Azevedo, C.A.V. de. (2016). The Assistat Software Version 7.7 and its use in the analysis of experimental data. African Journal of Agricultural Research, 11(39): 3733-3740. https://doi.org/10.5897/AJAR2016.11522.

Wielewicki, A.P.; Leonhardt, C.; Schlindwein, G. \& Medeiros, A.C.S. (2006). Proposta de padrões de germinação e teor de água para sementes dealgumas espécies florestais presentes na região sul do brasil. Revista Brasileira de Sementes, 28(3): 191-197. https://doi.org/10.1590/S0101-31222006000300027. 